\title{
PROTEINATOR: Web-UI exploring repurposing hypotheses of PROTEIN InhibiTORs based on protein interactions
}

\author{
Santhosh Tangadu, ${ }^{\dagger, \ddagger}$ Susmitha Shankar, ${ }^{\dagger, \ddagger}$ Bhaskaram V. Varanasi, ${ }^{\dagger}$ and \\ Prashanth Athri*,† \\ $\dagger$ Amrita Vishwa Vidyapeetham, Amrita School of Engineering, Bengaluru, Dept. of \\ Computer Science 83 Engineering, Bengaluru, India \\ $\ddagger$ Contributed equally \\ E-mail: prashanthathri@gmail.com
}

The authors have withdrawn this manuscript because in it's current stage, the work only identifies the plausible search space for new drug interactions, and is based on a biochemical phenomenon. Nonetheless, and as pointed out by 2 independent peer review processes from leading journals in this area, the results are of very little value without basic validation. We are a computer science department, and do not have the experimental setup to do the same. As we move forward, our goal is to collaborate with a life sciences (biophysical lab) group, and apply for funding to complete the validations for a few test cases before we re-submit this work and make it available to the rest of the community. Therefore, the authors do not wish this work to be cited as reference for the project. If you have any questions, please contact the corresponding author. 